\title{
Third order Intermodulation Distortion in Film Bulk Acoustic Resonators at Resonance and Antiresonance
}

\author{
Eduard Rocas ${ }^{1}$, Carlos Collado ${ }^{1}$, Jordi Mateu ${ }^{1,2}$, Humberto Campanella ${ }^{3}$, Juan M. O'Callaghan ${ }^{1}$ \\ ${ }^{1}$ Universitat Politècnica de Catalunya (UPC), Campus Nord, Barcelona, Spain \\ ${ }^{2}$ Centre Tecnològic de Telecomunicacions de Catalunya (CTTC), PMT, Castelldefels, Spain \\ ${ }^{3}$ Centro Nacional de Microelectrónica (CNM-CSIC), Campus UAB, Bellaterra, Spain
}

\begin{abstract}
This paper presents recent measurements and modeling of the third order intermodulation products of a Film Bulk Acoustic Resonator (FBAR), for a various values of frequency spacing between driving tones. The frequency dependence of voltage and current in the acoustic branch rules out a voltage-dependent nonlinearity. The results show different slopes at resonance and antiresonance, which are correctly adjusted by the model with a current dependent inductor and/or capacitor. The intermodulation distortion is found to be dependent on the frequency spacing between driving tones, indicating memory effects.
\end{abstract}

Index Terms - Thin-film bulk acoustic wave resonators, intermodulation, nonlinear characterization, resonance, antiresonance.

\section{INTRODUCTION}

Film bulk acoustic wave resonators (FBARs) allow to achieve very compact high-performance RF and microwave passive components [1]. The ever growing wireless market and a constantly increasing demand for spectrum are steadily pushing for more stringent requirements of such components. This is also the case of FBAR components and their nonlinear performance. In spite of the significance of the nonlinear effects in wireless communication systems [2], and the wellknown existence of nonlinearities in piezoelectric based devices such as FBARs [3],[4], there are not many published works focusing on the nonlinear characterization and modeling of the FBAR components and their impact in communication systems. Several physical phenomena occurring in a FBAR (piezoelectricity, elasticity, thermal effects, etc.) have a potential nonlinearity and may give rise to many nonlinear effects such as resonator detuning, saturation, and generation of harmonics and intermodulation distortion (IMD). The ability to predict all these effects with realistic and easy-to-use models would be of great help to designers and would facilitate the use of FBAR devices in future systems.

In contexts other than FBAR devices (superconducting and ferroelectric devices, for example), IMD has shown to be of great value to relate nonlinear phenomena with their measurable effects [5],[6],[7]. Following that guide, in this work we measure the IMD of an FBAR at resonance and antiresonance for a range of input power and for various values of frequency separation between the two tones feeding

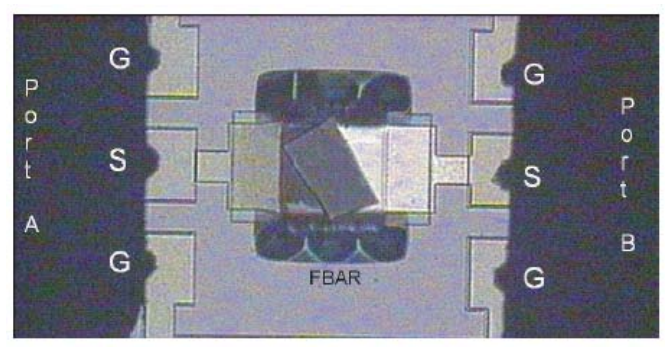

Fig. 1. FBAR tested in this work with coplanar transmission feed lines. The Area of the $1 \mu \mathrm{m}$ thick AlN membrane is 50x70 $\mu \mathrm{m}$.

the resonator. From the measurements we infer the basic features of a nonlinear version of the Butterworth Van Dyke model which we adjust to fit the measured data. The resulting model shows to be useful to predict the IMD at resonance and antiresonance. To our knowledge, there are no previous publications on nonlinear FBAR models able to account for IMD at these two resonant frequencies.

\section{TeSt DeVice AND LinEAR Modeling}

The FBAR tested in this work was implemented following the fabrication process described in [8]. This consists in a piezoelectric aluminium nitride (AIN) membrane $(50 \times 70 \mu \mathrm{m}$ and $1 \mu \mathrm{m}$ thick) sandwiched between two titanium/platinium layers and deposited on a silicon substrate. The geometry and dimensions of the FBAR tested were set to have resonant frequencies around $2.3 \mathrm{GHz}$. Embedded input and output coplanar waveguide (CPW) feeding ports were introduced on wafer in the test structure. A picture of the FBAR can be seen in Fig. 1.

\section{A. Frequency Response and Linear Circuit Elements}

Since the FBAR nonlinear performance strongly depends on its linear parameters, we first extracted these (or, equivalently, the FBAR equivalent circuit linear elements) from the smallsignal frequency response following the procedure described below.

We used an Agilent 8510 network analyzer to obtain the scattering parameters defining the FBAR frequency response from $1 \mathrm{GHz}$ to $2 \mathrm{GHz}$. An on-wafer thru-reflect-reflect-match (LRRM) calibration set was previously used to calibrate the 
TABLE I

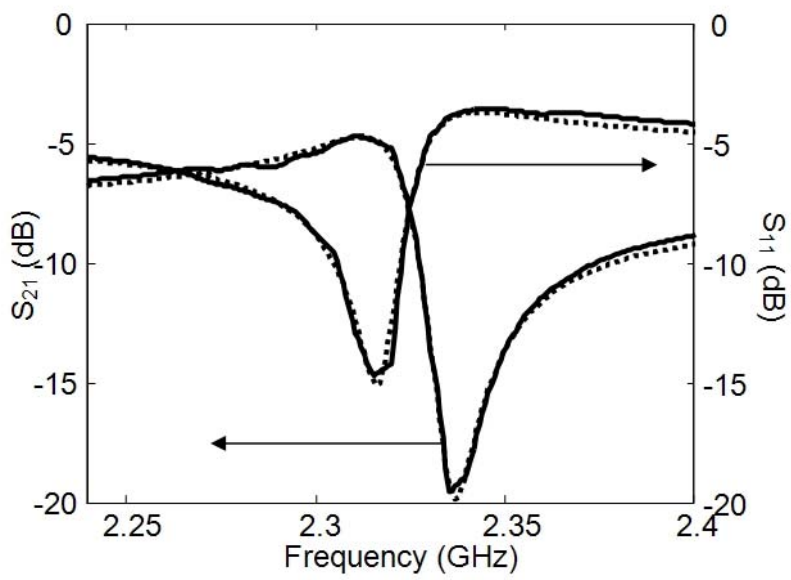

Fig. 2. In solid, measured $S_{21}$ and $S_{11}$. In dashed, simulated $S_{21}$ and $S_{11}$ modeling the FBAR with the circuit parameter of Fig.3 and Table I.

measurement set-up [9]. These measurements were performed for a power delivered by the network analyzer of $-10 \mathrm{dBm}$, which ensured the linear regime of the measured FBAR response.

Details of the frequency response, $S_{21}$ and $S_{11}$, are outlined in Fig. 2, in solid lines. Note that the frequency response exhibits a resonance frequency at $2.312 \mathrm{GHz}$ and anti-resonance frequency at $2.337 \mathrm{GHz}$

\section{B. Circuit Model}

The frequency response has been fitted using a modified Butterworth Van Dyke (MBVD) model. As in [8] additional capacitances $\left(C_{o x}\right)$ and resistances $\left(R_{s}\right.$ and $\left.R_{s u b}\right)$ have been introduced to consider the effects of the CPW feeding ports. The circuit model is outlined in Fig.3. A commercial software, [10], has been used to de-embed the circuit elements. Table I details the resulting parameters defining the circuit model, where the capacitances are in $\mathrm{fF}$, the inductance in $\mathrm{nH}$ and the resistances in ohms. Figure 2 also depicts, in dashed lines, $S_{21}$ and $S_{11}$ resulting from the circuit model response, showing very good agreement over the whole frequency range.

\section{IMD MEASUREMENTS IN FBAR}

We based the nonlinear characterization of the FBAR on performing intermodulation measurements. Due to the strong IMD produced by the FBAR, instead of using sophisticated setups that we used previously [5], we could use a simple one

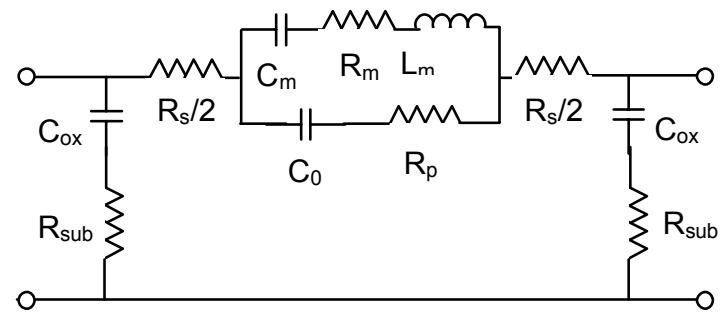

Fig. 3. Equivalent circuit model.
CIRCUIT PARAMETERS

\begin{tabular}{cccccccc}
\hline \hline $\mathbf{C}_{\mathbf{m}}$ & $\mathbf{R}_{\mathbf{m}}$ & $\mathbf{L}_{\mathbf{m}}$ & $\mathbf{C}_{\boldsymbol{o}}$ & $\mathbf{R}_{\mathbf{p}}$ & $\mathbf{R}_{\mathbf{s}}$ & $\mathbf{C}_{\text {ox }}$ & $\mathbf{R}_{\text {sub }}$ \\
\hline 10.2 & 14 & 464.5 & 551.1 & 11 & 60 & 438.3 & 220 \\
\hline \hline
\end{tabular}

in which the two sources synthesized at $f_{1}$ and $f_{2}$ are then combined to feed the nonlinear device, whose output is then driven to a spectrum analyzer to measure the magnitude of the intermodulation products at $2 f_{1}-f_{2}$ and $2 f_{2}-f_{1}$.

We systematically performed a set of IMD measurements at both resonance and antiresonance frequencies. In all these measurements we kept the two input tones balanced, that is $\mathrm{P}_{1}=\mathrm{P}_{2}$, where $\mathrm{P}_{1}$ and $\mathrm{P}_{2}$ are the powers of the tones at $\mathrm{f}_{1}$ and $\mathrm{f}_{2}$, respectively. The power driving the device was swept from $10 \mathrm{dBm}$ to $2 \mathrm{dBm}$ and measurements were carried out for $\Delta f$ (being $\Delta \mathrm{f}=\mathrm{f}_{2}-\mathrm{f}_{1}$ ) of $100 \mathrm{kHz}, 50 \mathrm{kHz}, 10 \mathrm{kHz}, 5 \mathrm{kHz} 1 \mathrm{kHz}$ and $100 \mathrm{~Hz}$.

Figure 4 shows the measured fundamental and IMD at resonance and antiresonance, respectively, for a tone spacing of $1 \mathrm{kHz}$. Circles and triangles represent the power at fundamental frequencies $f_{1}$ and $f_{2}$, respectively, and diamonds and squares represent the IMD at $2 f_{1}-f_{2}$ and $2 f_{2}-f_{l}$, respectively, all as a function of the power driving the FBAR, $P_{l}$. No IMD
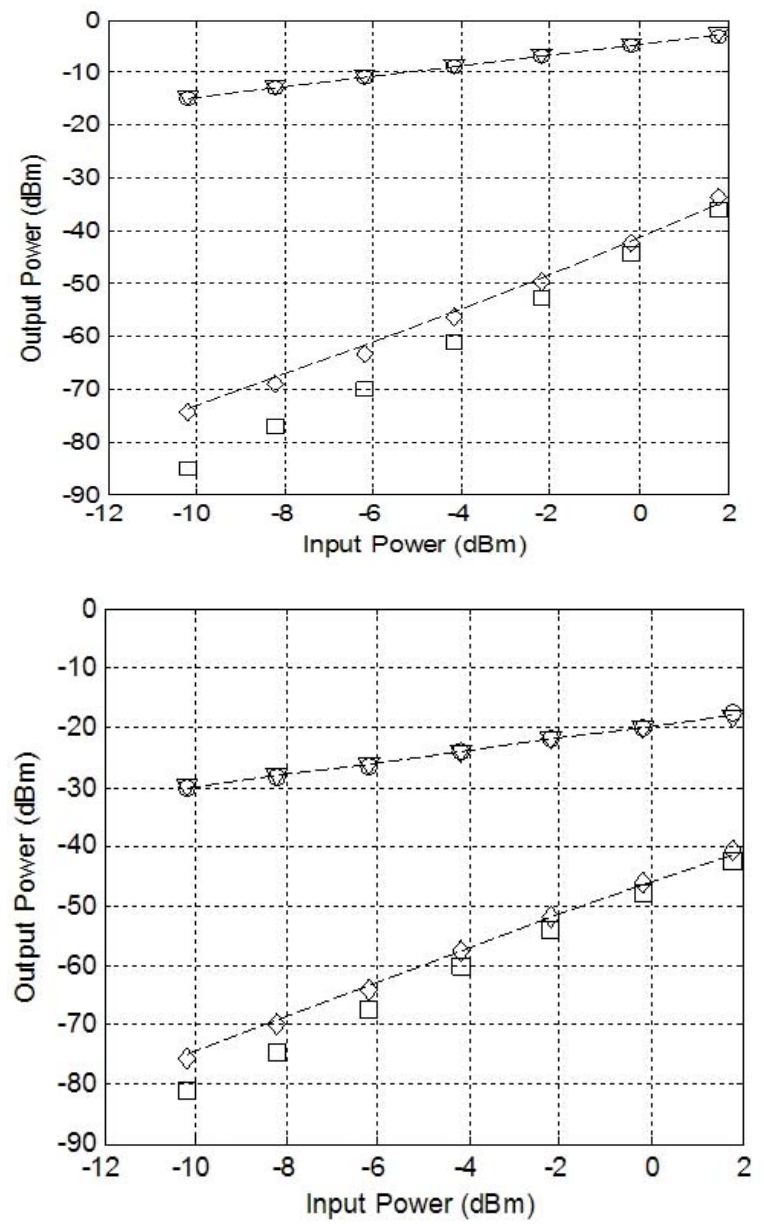

Fig. 4. Measured fundamental and IMD at resonance (top) and antiresonance (bottom) 


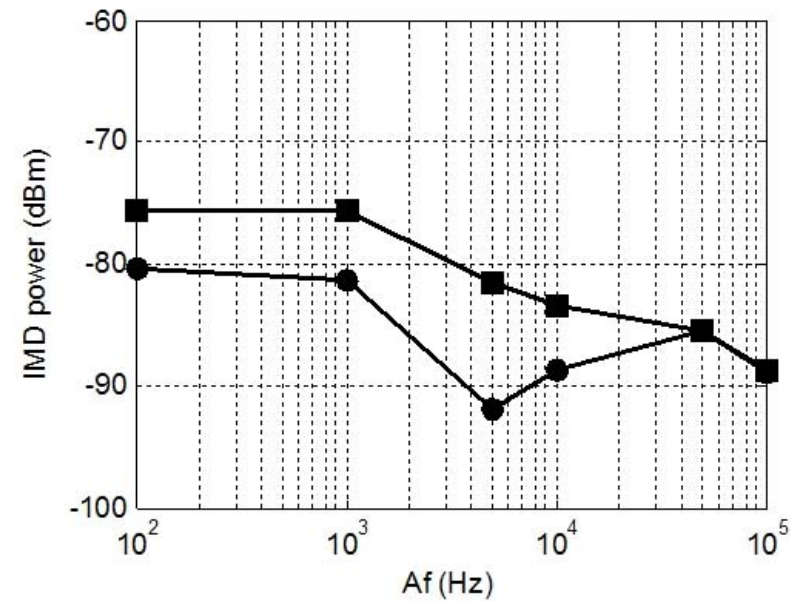

Fig. 5. IMD at $2 f_{1}-f_{2}$ (squares) and $2 f_{2}-f_{1}$ (circles) as a function of the frequency spacing $\Delta \mathrm{f}$, and for a fixed input power of $-10 \mathrm{dBm}$

response has been observed when the fundamental tones are set out of the resonant or antiresonant frequencies.

The power of the fundamentals follows a slope $1: 1$ in log$\log$ scale, over the whole range of measured power, for both cases (resonance and antiresonance), so neither detuning or saturation measurable effects affect the fundamental tones at these power levels (they might however affect IMD, as discussed later). On the other hand the slope of the IMD products is not the same for the resonant case than for the antiresonant one. Whereas for the resonant case the slope is slightly lower than 3 (in log-log scales), it is slightly higher than 3 for the antiresonant case.

Another important feature observed from the measured IMD is its dependence on the frequency spacing of the input tones $\Delta f$ and the asymmetries exhibited between the measured IMD at $2 f_{1}-f_{2}$ and $2 f_{2}-f_{1}$. Figure 5 shows the IMD at $2 f_{1}-f_{2}$ and $2 f_{2}-f_{1}$ for a fixed input power of $-10 \mathrm{dBm}$ as a function of the frequency spacing $\Delta f$. The increment of the IMD as the tone spacing decreases and the aforementioned asymmetry between the IMD might be due to several origins, but in general they might be attributed to the existence of memory effects with time constants similar to the period of the modulating signal (frequency $\Delta f$ ) in a two tone IMD experiment [11], [12].

\section{NONLINEAR MODELING APPROACH AND DisCUSSION}

This section presents a model that explains the nonlinear behavior of the IMD occurring at both resonances of the FBAR structure tested.

The nonlinear model proposed uses the linear circuit model presented in section II. B and assumes that the inductance $L_{m}$ and/or the capacitance $C_{m}$, depends on the current in the acoustic branch, as indicated in Fig.3. To analyze the effects of this assumption, Fig. 6 outlines the current in the acoustic branch and the voltage drop between node 1 and 2 of the

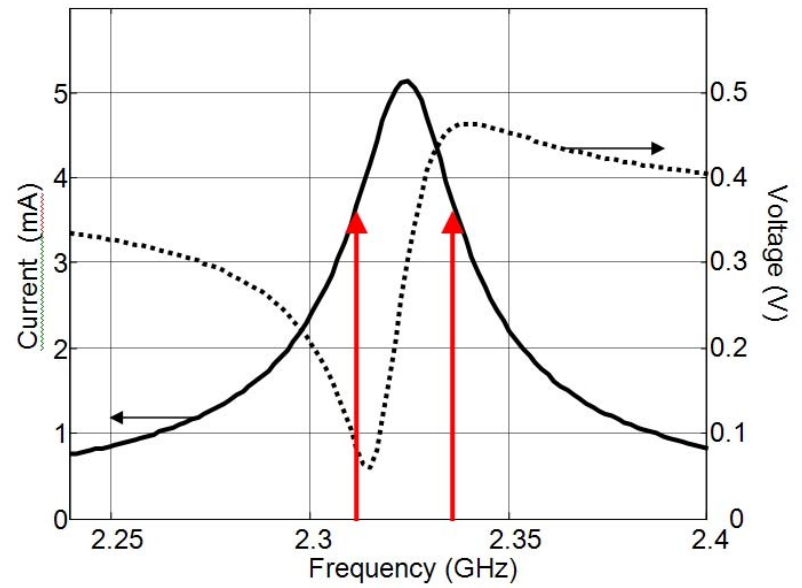

Fig. 6. Current flowing through the acoustic branch (solid line) and applied voltage to it (dotted line), as a function of frequency.

circuit model of Fig. 3, as a function of frequency. The arrows inserted in Fig. 6 indicate the frequencies of the resonance and anti-resonance. This shows that the current flowing through the acoustic branch of the circuit model is equal at both frequencies, and since the nonlinearities depend on this current, one could expect that the power of the generated IMD was similar in resonance and anti-resonance which is consistent with the measurements at low power levels (Fig. 4). By contrast, Fig. 6 rules out a voltage-dependent nonlinearity, since that would generate vastly different IMD at resonance and anti-resonance, due to the large difference in voltage at these two frequencies.

Having set that the nonlinearities should be currentdependent, the model can also explain the different slopes of the IMD graphs in Fig. 4 by a slight shift in the current response peak (Fig. 6) due to the dependence of $C_{m}$ or $L_{m}$ on current. A shift of the current frequency response curve to lower frequencies as the signal power is increased will tend to increase the slope of the IMD at resonance and decrease that of the IMD at antiresonance, while being experimentally unnoticeable to the fundamental signals at $f_{1}$ and $f_{2}$. This effect may be produced by a nonlinearity that makes $C_{m}$ increase with increased drive level and/or by a nonlinearity that also makes $L_{m}$ increase with increased drive level.

A current-dependent $C_{m}$ was assumed in [13] to fit the largesignal frequency response of quartz resonators. However, the dependence found in [13], set a decrease of $C_{m}$ with drive level, contrary what we have found in our case, where a dependence of the type $C(i)=C_{0}\left(1+\Delta C_{2} * i^{2}\right)$ with $\Delta \mathrm{C}_{2}=80 \mathrm{~A}^{-2}$ would fit the IMD curves at resonance and antiresonance throughout the whole range of input power used in the measurement (Fig. 4). Alternatively, one could set the nonlinearity in the inductor $L_{m}$ by using $L(i)=L_{0}\left(1+\Delta L_{2} * i^{2}\right)$ with $\Delta \mathrm{L}_{2}=28 \mathrm{~A}^{-2}$ and would obtain equivalent results. The dashed lines in Fig. 4 are obtained by using this equation. 
In [14] the nonlinear impedance of several piezoelectric resonators is analyzed considering only the nonlinear elasticity of the piezoelectric material. Contrary to what is found in [13], all the measurements in [14] show an increase in the reactive part of the impedance when $i$ increases. This is consistent with an increase of $\mathrm{C}$ and/or $\mathrm{L}$ with the current that would justify the IMD measurements presented here. (Note that, the increase in $\mathrm{C}$ will diminish the absolute value of the reactance, but will produce the reactance increase mentioned previously, since $\mathrm{C}$ affects the negative part of the reactance.)

This simple picture of the FBAR nonlinear response might be useful to explain the IMD at both resonance and antiresonance frequencies for a wide range of powers. Additional considerations will have to be made to take into account memory effects and to jointly study IMD with other nonlinear effects such as saturation and detuning.

\section{ACKNOWLEDGMENT}

This work was partially supported by the Spanish Government (CICYT) under Grant TEC-2006-13248-C0402/TCM. E. R and J. M thank the support from BES-200716775 and RyC-001125.

\section{REFERENCES}

[1] K.M. Lakin; G.R. Kline; K.T. McCarron, "High Q microwave acoustic resonators and filters," Microwave Symposium Digest, 1993., IEEE MTT-S International , vol., no., pp.1517-1520 vol.3, 1993

[2] Pedro and Carvalho, "Intermodulation Distortion in Microwave and Wireless Circuits," Artech House: Norwood, Mass., 2003.

[3] Kaltenbacher, B.; Hofer, M.; Kaltenbacher, M.; Simkovics, R.; Lerch, R., "Identification of material nonlinearities in piezoelectric ceramics," Ultrasonics, 2003 IEEE Symposium on , vol.1, no., pp. 358-361 Vol.1, 5-8 Oct. 2003
[4] S P Joshi, "Non-linear constitutive relations for piezoceramic materials", Smart Materials and Structures. March 1992 Volume: 1 Start Page: 80

[5] J. Mateu, J. C. Booth, S. A. Schima "Frequency Tuning and Spurious Signal Generation in Ferroelectric Thin-film transmission lines" IEEE Trans. On Microwave Theory and Techniques, vol. 55, no. 2, February, 2007

[6] C. Collado, J. Mateu, J. M. O'Callaghan, "Analysis and Simulation of the Effects of Distributed nonlinearities in microwave superconducting devices" IEEE Trans. On Appl. Supercond. , vo. 15, no.1, pp. 26-39, March 2005

[7] J. Mateu, J. C. Booth, B. H. Moeckly, "Wideband Nonlinear Response of High-Temperature Superconducting Thin Films From transmission line measurements", IEEE Trans. On Microwave Theory and Techniques, vol. 55, no. 7, July, 2007

[8] H. Campanella, P. Nouet, P. de Paco, A. Uranga, N. Barniol, and J. Esteve, "Automated on-Wafer Extraction of EquivalentCircuit Parameters in Thin-Film Bulk Acoustic Wave Resonators and Substrate" Microwave And Optical Technology Letters Vol. 50, No. 1, pp. 4-7, 2008

[9] F. Purroy, L. Pradell, "New theoretical analysis of the LRRM calibration technique for vector network analyzers," IEEE Trans. On Instrumentation and Measurement, Vol. 50, No.5, pp. 1307-1314, 2001.

[10] Advanced Design Systems (ADS)

[11] Ngoya, E.; Le Gallou, N.; Nebus, J.M.; Buret, H.; Reig, P., "Accurate RF and microwave system level modeling of wideband nonlinear circuits," Microwave Symposium Digest., 2000 IEEE MTT-S International , vol.1, no., pp.79-82, 2000

[12] N. Borges de Carvalho, J.C. Pedro, "A Comprehensive Explanation of Distortion Sideband Asymmetries", IEEE Trans. On Microwave Theory and Techniques, vol., 50, no. 9, 2002.

[13] J. Nosek, "Drive Level Dependence of the Resonant Frequency in BAW Quartz Resonators and His Modeling", IEEE Trans. On Ultrasonics, Ferroelectrics and Frequency Control, Vol. 46, no. 4, July 1999.

[14] R. Perez and A. Albareda "Analysis of nonlinear effects in a piezoelectric resonator", J. Acoust. Soc. Am. Vol. 100, no. 6, pp 3561-3569, 1996 